\title{
Nonlinear Feedback Excitation for System Interrogation by Bifurcation Morphing
}

\author{
Shih-Hsun Yin* \\ National Taipei University of Technology, \\ Taipei 106, Taiwan, Republic of China \\ and \\ Bogdan I. Epureanuı̇ \\ University of Michigan, Ann Arbor, Michigan 48109-2125 \\ DOI: $10.2514 / 1.35028$
}

\begin{abstract}
This paper demonstrates a novel system-interrogation method based on observing the morphing of bifurcations and the postbifurcation dynamics through both experimental and numerical methods. A sensing cantilever beam is built with lead-zirconate-titanate patches symmetrically bonded to both sides of its root. A desired bifurcation in the dynamics of the beam can be induced by applying a nonlinear feedback excitation to the beam. The nonlinear feedback excitation requires the active measurement of the dynamics and a feedback loop, and it is generated by applying the voltage (resulting from a nonlinear feedback) to the piezoelectrode. Also, a finite element model is used to design the nonlinear feedback excitation and to predict the response of the sensing beam. Numerical simulations and experiments are performed and compared that demonstrate the effectiveness and high level of sensitivity of the novel approach to detect very small amounts of mass added at the tip of the beam.
\end{abstract}

\section{Nomenclature}

$\begin{array}{ll}a & =\text { linear gain } \\ \mathbf{C} & =\text { damping matrix in a finite element model } \\ c & =\text { damping in a one-degree-of-freedom model } \\ \mathbf{F} & =\text { forcing vector } \\ \mathbf{K} & =\text { stiffness matrix in a finite element model } \\ k & =\text { stiffness in a one-degree-of-freedom model } \\ \mathbf{M} & =\text { mass matrix in a finite element model } \\ m & =\text { mass in a one-degree-of-freedom model } \\ p_{i} & =\text { parameters of the nonlinear controller } \\ V & =\text { voltage applied to lead-zirconate-titanate patches } \\ v & =\text { velocity at the tip of the sensing beam } \\ \mathbf{X} & =\text { state-space vector in a finite element model } \\ \alpha, \beta & =\text { structural damping characteristics } \\ \gamma, \delta & =\text { nonlinear gains } \\ \xi & =\text { damping ratio } \\ \Omega & =\text { frequency of the harmonic component of the excitation }\end{array}$

\section{Introduction}

$\mathbf{V}$ IBRATION-BASED techniques are commonly used for system interrogation in applications such as structural health monitoring $[1,2]$. Such methods monitor changes in vibratory characteristics of a structure, which reflect damage. Several of these techniques use subspace identification and updating [3-6], wavelet analyses [7,8], and Ritz vectors [9,10]. Although there have been numerous studies showing that changes in observed linear features can be used to detect the presence of damage, the low sensitivity of these features (to damage) limits the applicability of such methods. To increase sensitivity to parameter variations indicative of damage,

Received 7 October 2007; revision received 25 January 2008; accepted for publication 16 February 2008. Copyright @ 2008 by Shih-Hsun Yin and Bogdan I. Epureanu. Published by the American Institute of Aeronautics and Astronautics, Inc., with permission. Copies of this paper may be made for personal or internal use, on condition that the copier pay the $\$ 10.00$ per-copy fee to the Copyright Clearance Center, Inc., 222 Rosewood Drive, Danvers, MA 01923; include the code 0001-1452/08 \$10.00 in correspondence with the CCC

*Assistant Professor, Department of Civil Engineering, Number 1, Section 3, Chung-Hsiao East Road; shihhsun@ntut.edu.tw.

${ }^{\dagger}$ Associate Professor, Department of Mechanical Engineering, 2350 Hayward Street; epureanu@umich.edu. Member AIAA. the concept of sensitivity enhancing control [11-13] and designed impedance techniques [14-16] were proposed through linear feedback control applied to a structure. Such approaches reduce modal frequencies to enhance their sensitivity to changes in stiffness. Other studies have exploited nonlinearities to enhance sensitivity. For example, linear systems subjected to chaotic excitation [17-21] and (nonlinear or) chaotic systems (with or without excitation) 24] have been explored to show that the use of nonlinearity holds a great potential for damage detection. Furthermore, enhancing nonlinearity within linear or weakly nonlinear systems by means of nonlinear feedback excitation has been demonstrated computationally to provide significant advantages such as increased sensitivity $[25,26]$

In the present work, we apply a novel detection method for identifying small parameter changes in a smart structure. The method is based on observing the morphing of bifurcations and the dynamics in the postbifurcation regime. For most structures, dissipative mechanisms balance the external excitation such that the dynamics evolve onto an invariant manifold of the state space (the attractor). Examples of attractors of the dynamics of a structure are stable fixed points, stable limit cycles, and strange attractors (for chaotic systems). A qualitative change in the dynamics (referred to as a bifurcation) may happen as parameters are varied. For example, fixed points or limit cycles can be destroyed or created or their stability can change when bifurcations occur. The key idea of the method herein is to actively change the original stability of a fixed point (equilibrium state) or the dynamic response of a system (structure) and to create a new stable fixed point, a limit cycle, or a more complex dynamic response by applying a nonlinear feedback excitation to the structure. The controller parameters (gains) of the nonlinear feedback excitation are manipulated to interrogate the system by identifying and characterizing bifurcation points. The main concept and key theoretical investigations of this approach have been presented by the authors [27,28].

In contrast, the goal of this paper is to test and validate the proposed method in an experimental way. Therefore, a smart structure composed of a cantilever beam in which two leadzirconate-titanate (PZT) piezoelectric patches are symmetrically bonded to both sides of the root of the beam is built as shown in Fig. 1 . Piezoelectric actuators and sensors are currently used as elements of intelligent structures [29] and are widely exploited for active vibration suppression and structural health monitoring in smart 


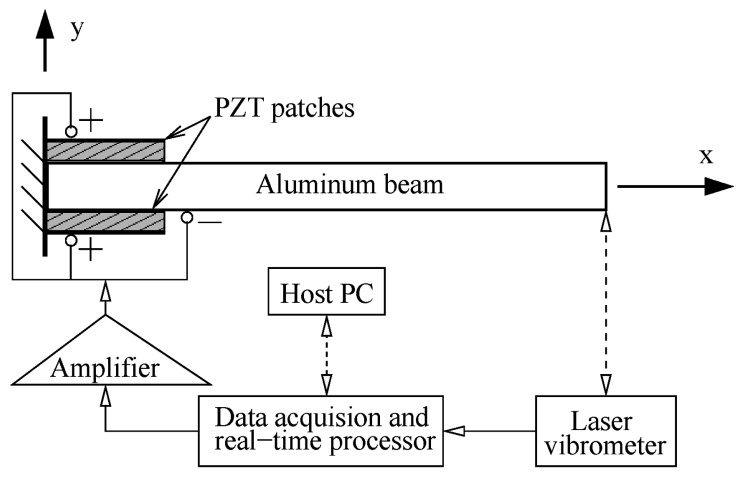

Fig. 1 Schematic of the experimental setup.

structures [30-33]. In this work, several complex dynamics of the beam can be induced by applying a designed nonlinear feedback excitation to the beam. First, the motion of the tip of the beam is measured using a laser vibrometer, and the measured signal is used in a real-time processor to which a designed nonlinear control circuit is uploaded from a host computer. Finally, the output voltage from the real-time processor is applied to the PZT patches to excite the beam.

The actual bifurcation point of the dynamics can be determined by sweeping the values of the gains in the nonlinear control circuit and recording the values at which bifurcations occur. Also, a finite element model of the sensing beam is used to design the closed-loop controller and to predict the bifurcation point and the postbifurcation dynamics. Results from numerical simulations and experiments are compared to demonstrate the novel approach and to evaluate its effectiveness and robustness.

\section{System Interrogation Methodology}

In this work, a system-interrogation method based on observing the onset of bifurcations and the emerging dynamics in the postbifurcation regime is developed. The controller parameters for the nonlinear feedback excitation are manipulated to interrogate the system by identifying the bifurcation point. Consider, for clarity, the very simple example of a single-degree-of-freedom oscillator forced by a specific feedback excitation. The governing equation for this simple oscillator can be expressed as

$$
m \ddot{x}+c \dot{x}+k x=a x-\delta x^{3}
$$

where $m, c$, and $k$ are mass, damping, and stiffness parameters; $a$ and $\delta$ are the linear and nonlinear controller parameters; and all of these parameters are assumed to be positive. A supercritical pitchfork bifurcation occurs when $a$ equals $k$, as shown in Fig. 2. Thus, the stiffness $k$ could be directly interrogated by varying the value of $a$ and observing the onset of the bifurcation for this special case. Hence, the change in stiffness due to damage can be detected by distinguishing between the bifurcation points for a healthy and a damaged system. Also, the type of bifurcation and the dynamics in

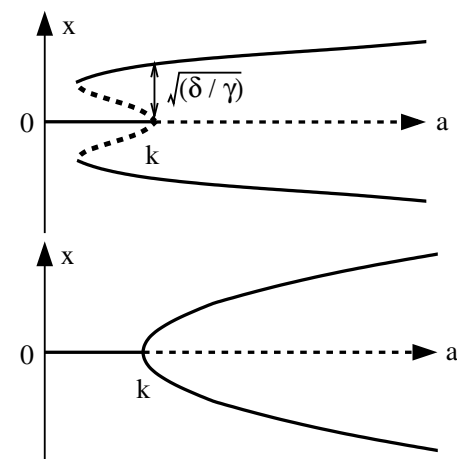

Fig. 2 Fixed points of an oscillator (forced by nonlinear feedback excitation) undergoing subcritical (top) or supercritical (bottom) pitchfork bifurcations. the postbifurcation regime depend on the form of the nonlinear feedback. For example, if a different kind of nonlinear feedback is used (e.g., $\delta x^{3}-\gamma x^{5}$ instead of $-\delta x^{3}$ ) in Eq. (1), then a different type of bifurcation (i.e., subcritical) may occur, as shown in Fig. 2.

Also, in general, the origin of the state space is a stable fixed point (equilibrium state) for most autonomous systems. Hence, in its simplest form, the key idea of the proposed method is to actively change the stability of the fixed point at the origin (by tuning the values of the controller parameters) and to design the nonlinear feedback such that a new stable fixed point or a limit cycle emerges close to the origin. These bifurcations, caused by the designed nonlinear feedback excitation for this simple scenario, are pitchfork or Hopf bifurcations. Next, changes in bifurcation boundaries (i.e., changes in the loci of bifurcation points) in the space of the controller parameters can be exploited to identify changes in structural parameters caused by damage [27]. In this paper, in addition to the autonomous feedback, a time-dependent force is added to the nonlinear feedback excitation. The resulting system is unlike autonomous systems in which pitchfork or Hopf bifurcations occur. Because of the explicit time dependence of the excitation, the system exhibits more complex dynamics when the controller parameters are varied. Such qualitative or quantitative changes in dynamics are sensitive to very small changes in structural parameters. Therefore, observing the changes in bifurcation diagrams (and, in particular, the morphing of bifurcation boundaries caused by a nonlinear feedback combined with a time-dependent force) is beneficial for detecting incipient damage. In comparison with pitchfork and Hopf bifurcation boundaries that can be analytically predicted by exploring the eigenvalues of the Jacobian matrix at the origin [27], investigating the changes in dynamics of the nonautonomous nonlinear system relies primarily on numerical analysis. Whether numerically simulating or experimentally implementing the proposed approach, the bifurcation diagrams and the locus of bifurcation points for detecting changes in structural parameters can be obtained by varying the values of the controller parameters in the numerical model or in the control circuit in the experimental setup.

\section{Experimental Setup and Procedure}

The sensing cantilever beam is composed of an aluminum-alloy beam with two PZT patches (supplied by Piezo Systems, Inc.: material type 5H4E, industry type 5H, and U.S. Navy type VI) symmetrically bonded on its surfaces near the clamp location using an epoxy adhesive, as shown in Fig. 1. The dimensions and properties of the beam and PZT patches [34] (some measured directly and others provided by Piezo Systems, Inc.) are given in Table 1 . The two PZT patches have the same orientation of their polarization, such that the bimorph configuration can generate larger deflections.

A laser vibrometer is used to measure the velocity at the tip of the beam. In addition to the laser vibrometer (manufactured by Polytec), there are several apparatus used for experiments, including a function generator (manufactured by Agilent), an amplifier (manufactured by Khron-Hite), a laptop, and a real-time processor (manufactured by Tucker-Davis Technologies). The real-time processor is used for data acquisition and signal processing and can be controlled through a computer interface. Therefore, the controller circuits can be coded offline or online and then uploaded from the host laptop to the realtime controller. The output analog signal is generated through the circuits as a nonlinear function of the measured signal combined with

Table 1 Dimensions and properties of the sensing beam

\begin{tabular}{lcc}
\hline \hline & Aluminum beam & PZT patch \\
\hline Length, mm & 280 & 60 \\
Width, mm & 15 & 15 \\
Thickness, mm & 1.27 & 1 \\
Young's modulus, GPa & 68.9 & 62 \\
Density, kg/m ${ }^{3}$ & 2660 & 7800 \\
Poisson's ratio & 0.33 & 0.31 \\
Piezoelectric constant, $10^{-9} \mathrm{~mm} / \mathrm{V}$ & - & -300 \\
\hline \hline
\end{tabular}


a time-dependent function. Meanwhile, the measured signal can be saved in a buffer of the real-time controller and then downloaded to the host laptop for postprocessing (such as creating bifurcation diagrams and tracking bifurcation points).

Overall, a nonlinear feedback excitation is formed by a feedback loop consisting of sending the analog signal measured from the laser vibrometer into the real-time processor, processing this signal via a designed circuit in the processor, amplifying the analog output signal from the processor, and then applying the amplified voltage to the PZT patches to excite the beam. The net effect of the applied voltage is to introduce a torque at the ends of the piezopatches, causing the sensing beam to bend. The actual bifurcation point of the dynamics can be determined by varying the values of the parameters used in the controller and recording the values at which the dynamics change qualitatively.

To explore the active bifurcation-based method for identifying parameter variations exploiting active bifurcation morphing, masses (made of wax) of different weights are attached to the sensing beam to create parameter variations in the system. The reason for using mass variations is that these variations are easily quantifiable, reproducible, and reversible. Furthermore, many applications use mass detectors, such as a class of biological and chemical sensors, and also certain corrosion detectors, which identify corrosion by detecting loss of mass in a structure.

\section{Finite Element Modeling}

The purpose of modeling the sensing beam is to develop a mathematical description for understanding and controlling the system behavior. The finite element method has been shown to be an effective tool for modeling smart structures $[31,35]$. In this study, the governing equation of the sensing beam is obtained using both regular beam elements and piezobeam elements (a composite beam composed of two piezoelectric layers perfectly bonded to a regular beam). Therefore, the mass and stiffness matrices of the sensingbeam model include the contribution of the mass and stiffness of the PZT patches. The elemental mass and stiffness matrices are derived from the kinetic and strain energy equations of an element based on Euler-Bernoulli beam theory. Then the global mass and stiffness matrices $\mathbf{M}$ and $\mathbf{K}$ are formed, and a Rayleigh damping matrix $\mathbf{C}$ is used in the modeling as

$$
\mathbf{C}=\alpha \mathbf{M}+\beta \mathbf{K}
$$

where $\alpha=2 \xi \omega_{1} \omega_{2} /\left(\omega_{1}+\omega_{2}\right), \beta=2 \xi /\left(\omega_{1}+\omega_{2}\right), \omega_{1}$ and $\omega_{2}$ are the first and second modal frequencies, and $\xi$ is the specified damping ratio. Also, based on the assumption of perfect bonding layer (i.e., zero-thickness layer that allows no relative motion between the PZT and aluminum components) and effectively transferred shear force [29], the moment exerted at a node of a piezobeam element due to the applied voltage can be expressed as

$$
M_{0}=\frac{E_{b} t_{b} E_{p} t_{p}}{E_{b} t_{b}+6 E_{p} t_{p}} A_{b} \frac{d_{31} V}{t_{p}}
$$

where $E_{b}$ and $E_{p}$ are Young's modulus of the aluminum beam and of the PZT patches, $t_{b}$ and $t_{p}$ are the thickness of the aluminum beam and of the PZT patches, $A_{b}$ is the cross-sectional area of the aluminum beam, $d_{31}$ is the piezoelectric constant of the PZT patches, and $V$ is the voltage applied to the PZT patches. The applied voltage can be a combination of a nonlinear function of the measured signal and any time-dependent functions through a feedback loop. Thus, the nonlinear feedback excitation $\mathbf{F}$ can be modeled by assembling all (elemental) nodal forces and by using the specific form of the nonlinear control circuit (embedded in the real-time processor) and the gain of the amplifier. Finally, the equation of motion for the discretized sensing-beam model subjected to the nonlinear feedback excitation can be expressed as

$$
\mathbf{M} \ddot{\mathbf{x}}+\mathbf{C} \dot{\mathbf{x}}+\mathbf{K} \mathbf{x}=\mathbf{F}
$$

where $\mathbf{x}$ is the vector of nodal displacements. Equation (4) can be expressed in state-space representation by introducing a new vector $\mathbf{v}=\dot{\mathbf{x}}$ as follows:

$$
\dot{\mathbf{X}}=\mathbf{A} \mathbf{X}+\mathbf{B F}
$$

where

$$
\begin{gathered}
\mathbf{A}=\left[\begin{array}{cc}
0 & I \\
-\mathbf{M}^{-1} \mathbf{K} & -\mathbf{M}^{-1} \mathbf{C}
\end{array}\right], \quad \mathbf{B}=\left[\begin{array}{c}
0 \\
\mathbf{M}^{-1}
\end{array}\right] \\
\mathbf{X}=\left[\begin{array}{ll}
\mathbf{x} & \mathbf{v}
\end{array}\right]^{T}, \quad \mathbf{F}=\mathbf{G} \mathbf{X}+\overline{\mathbf{F}}(\mathbf{X})+\overline{\mathbf{F}}(t)
\end{gathered}
$$

Herein, the nonlinear feedback excitation $\mathbf{F}$ is a function of $\mathbf{x}, \dot{\mathbf{x}}$, and $t$ and consists of nonlinear feedback $\overline{\mathbf{F}}(\mathbf{X})$, a time-dependent function $\overline{\mathbf{F}}(t)$, and linear feedback $\mathbf{G X}$, where $\mathbf{G}$ is a linear gain matrix. The dynamics of the sensing beam forced by nonlinear feedback excitation can be obtained by solving Eq. (5) using any timemarching scheme. To generate a bifurcation diagram computationally, the values of the controller parameters used in the nonlinear feedback excitation are varied, and the dynamics of the sensing beam are recorded from numerical integrations done for each set of parameters.

\section{Nonlinear Feedback Excitation Design}

Two important questions in the experimental setup used herein are as follows:

1) What combination of nonlinear functions (of the measured signal) should be used for bifurcation morphing?

2) What time-dependent functions should be used in the controller such that small changes in mass lead to large changes in the bifurcation boundary?

The design of the controller cannot be as simple as in most investigations using numerical simulation. As discussed in Sec. IV, the linear model of the sensing beam is obtained easily by the finite element method. In contrast, creating an appropriate form of nonlinear feedback excitation $\mathbf{F}$ is more challenging, especially experimentally.

The controller designed for the sensing beam draws inspiration from well-known driven dissipative flows. Specifically, the form of nonlinear feedback excitation can be defined by mimicking a driven van der Pol oscillator. To that aim, consider first that the cantilever beam may be approximated as a one-degree-of-freedom system (e.g., corresponding to the first Galerkin mode). The governing equation of a one-degree-of-freedom van der Pol oscillator can be expressed as

$$
\ddot{x}+x=b\left(1-x^{2}\right) \dot{x}+P \sin \Omega t
$$

where $b, P$, and $\Omega$ are related to the controller parameters. This oscillator can exhibit diverse dynamics when different parameters are chosen. The main causes for the rich dynamics are nonlinearity and the harmonic force. However, the nonlinear form in Eq. ( $\underline{6})$ cannot be directly used in nonlinear feedback excitation, because the measured signal in our experiment is only the velocity of one point on the beam, rather than both displacement and velocity. Therefore, Eq. (6) requires modifications. First, Eq. (6) can be integrated with respect to time to obtain

$$
\dot{x}+\int x \mathrm{~d} t=b x-\frac{b}{3} x^{3}-\frac{P}{\Omega} \cos \Omega t+c_{1}
$$

where $c_{1}$ is a constant of integration. Then, by introducing $y=\int x \mathrm{~d} t-c_{1}$, Eq. (7) can be written as

$$
\ddot{y}+\varepsilon \dot{y}+y=(b+\varepsilon) \dot{y}-\frac{b}{3} \dot{y}^{3}-\frac{P}{\Omega} \cos \Omega t
$$

where $\varepsilon$ characterizes the structural damping in the sensing beam. The dynamic of the new state $y$ is qualitatively similar to the dynamic of the state $x$ in Eq. (6) if $x$ is smooth. Thus, $y$ can also exhibit various dynamics when different parameters are selected. In Eq. ( $\underline{8}$ ), the lefthand side represents one mass-spring system, and the right-hand side can be regarded as the nonlinear feedback excitation applied to the 
Table 2 First four frequencies (in hertz) for the sensing beam with no mass and with $20 \mathrm{mg}$ added at the tip

\begin{tabular}{lccc}
\hline \hline & No mass added & 20-mg mass added & Relative difference, \% \\
\hline Mode 1 & 20.5589 & 20.4877 & 0.3460 \\
Mode 2 & 123.2820 & 122.8804 & 0.3258 \\
Mode 3 & 321.9955 & 321.0694 & 0.2876 \\
Mode 4 & 563.9966 & 562.5962 & 0.2483 \\
\hline
\end{tabular}

mass. One of the most important aspects of this design is that the nonlinear feedback excitation depends only on the velocity $\dot{y}$ and yet causes diverse dynamics. Based on this exploration, we specify the applied voltage $V$ in Eq. (3) as

$$
V=p_{1} v+p_{2} v^{3}+p_{3} \sin \Omega t
$$

where $v$ is the velocity measured using the laser vibrometer at the tip of the beam, and $p_{1}, p_{2}, p_{3}$, and $\Omega$ are the parameters of the nonlinear feedback excitation.

\section{Results}

Consider computational results first. Different parameter variations can be applied computationally; in particular, consider adding no mass, 10 , and $20 \mathrm{mg}$ at the tip of the sensing beam. Table 2 shows the first (lowest) four frequencies for the sensing beam with no mass and with $20 \mathrm{mg}$ mass added at the tip. The changes in these four frequencies due to a small amount of added mass are very small. Hence, most usual linear vibratory characteristics are not sensitive enough to this small change in mass. In contrast, the proposed approach takes advantage of nonlinearity resulting from the designed nonlinear feedback excitation. Herein, the values of the parameters $p_{2}, p_{3}$, and $\Omega$ are fixed and specified as 4000,1 , and 128.81 . The value of the parameter $p_{1}$ is varied from 670 to 750 with an increment of unity. The reason for choosing the parameter $p_{1}$ as the bifurcation parameter is to destabilize the original linear damping in the structure and to create negative linear damping. In addition, the nonlinear damping (designed in the feedback excitation) allows the response of the structure to remain bounded, which is the same situation as for the driven van der Pol oscillator modeled in Eq. (8) (where $b<0$ ).

Figure 3 shows the bifurcation diagrams for the cases in which different amounts of mass (no mass, 10, and $20 \mathrm{mg}$ ) are added at the tip of the beam. Compared with the low sensitivity of linear frequencies, the differences between the bifurcation diagrams are so significant that a very small amount of mass (such as $10 \mathrm{mg}$ ) can be easily detected by monitoring the value of $p_{1}$ (for which the minimum local amplitude is obtained). This enhanced sensitivity is the key feature for the proposed approach. When the value of the controlled parameter $p_{1}$ is less than that for the Hopf bifurcation point, nonlinear effects are small [for small amplitudes, $p_{3}$ of the harmonic part of the excitation in Eq. (9)] and the steady-state response of the sensing beam are dominated by the linear behavior (i.e., a harmonic response of frequency $\Omega$ ). Such dynamics are shown in Fig. 4 (left). Hence, the response exhibits only one local amplitude maximum, shown as one point in the bifurcation diagrams (e.g., Fig. 3). The amplitude of this harmonic response can be estimated using Eq. ( $\underline{8}$ ) (where $b+\varepsilon=p_{1}$ ) as

$$
\begin{aligned}
A_{l} & =\frac{P / \Omega}{\sqrt{\left(1-\Omega^{2}\right)^{2}+\left(\varepsilon-p_{1}\right)^{2} \Omega^{2}}} \\
& =\frac{1}{\sqrt{\left(1-\Omega^{2}\right)^{2} \Omega^{2} / P^{2}+\left(\varepsilon-p_{1}\right)^{2} \Omega^{4} / P^{2}}} \\
& =\frac{1}{\sqrt{c_{1}+c_{2}\left(c_{3}-p_{1}\right)^{2}}}
\end{aligned}
$$

where $c_{1,2,3}$ are constants that characterize the sensing beam and the harmonic part of the excitation, and they are dependent on $\Omega$ and $P$ [which is related to the parameter $p_{3}$ of the applied voltage $V$ given in Eq. (9)].

After $p_{1}$ passes the bifurcation point, the negative damping created by the controller surpasses the existing damping in the sensing beam, and the system loses its linear stability. Thus, in this regime, the dynamics are the combination of an emerging limit cycle (periodic motion of frequency denoted by $\Omega_{n}$ ) born by undergoing a Hopf bifurcation and the response to the harmonic component of the excitation (of frequency $\Omega$ ). These two periodic oscillations have distinct frequencies (in general, $\Omega \neq \Omega_{n}$ ), and that leads to various local amplitude maxima, shown as many points on a vertical line segment in the bifurcation diagrams (e.g., Fig. 3). The amplitude of the part of the response that is mainly caused by the harmonic component of the excitation can be approximated by using Eq. (10). The amplitude of the part of the response that is caused by the limitcycle oscillation born through the Hopf bifurcation grows as a square root of the distance from $p_{1}$ to the bifurcation point [36] and can be estimated as

$$
A_{n}=c_{4} \sqrt{p_{1}-c_{5}} \text { for } p_{1} \geq c_{5}
$$

where $c_{4}$ is a constant that depends on the nonlinear terms of the excitation (i.e., $p_{2}$ ), and parameter $c_{5}$ represents the critical value of $p_{1}$ (i.e., the bifurcation point).

In the post-Hopf-bifurcation regime, the response can be qualitatively estimated as a combination of two oscillations: one of amplitude $A_{l}$ and frequency $\Omega$ and one of amplitude $A_{n}$ and frequency $\Omega_{n}$. When the frequencies of these oscillations have close values, the dynamics that occur are similar to a beating phenomenon. Such dynamics are shown in Fig. 4 (right). When the frequencies and the amplitudes $A_{l}$ and $A_{n}$ both have close values, then a very small (close to zero) local amplitude maximum occurs. Such dynamics are shown in Fig. 4 (center). In particular, the envelope of the full response (interpreted as two overlapping oscillations) can be expressed using Eqs. (10) and (11) as a lower envelope/curve $E_{L}$ and an upper envelope/curve $E_{U}$, both functions of $p_{1}$, given by
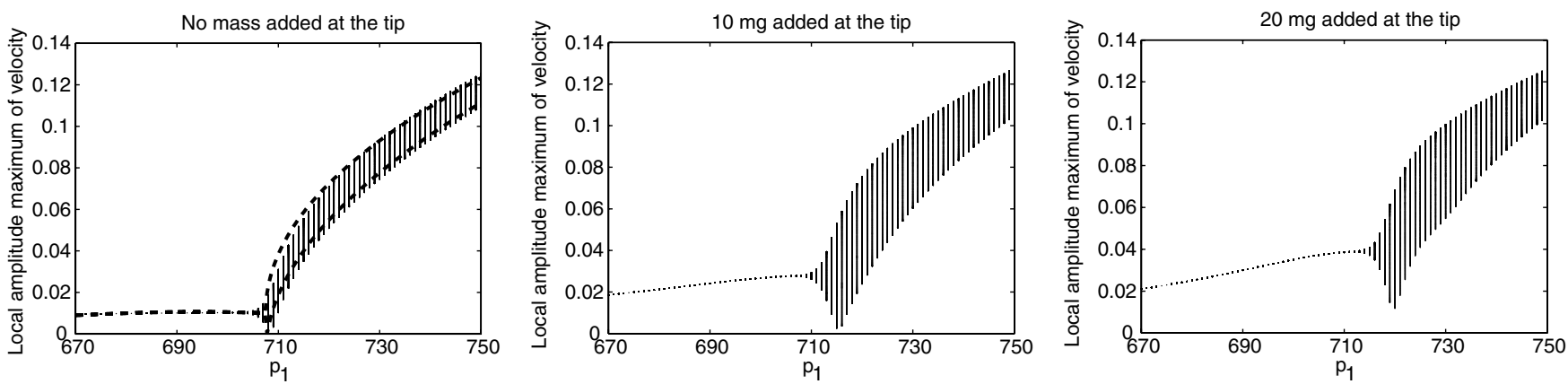

Fig. 3 Bifurcation diagrams for the cases in which different amounts of mass are added at the tip of the beam (computational results). 

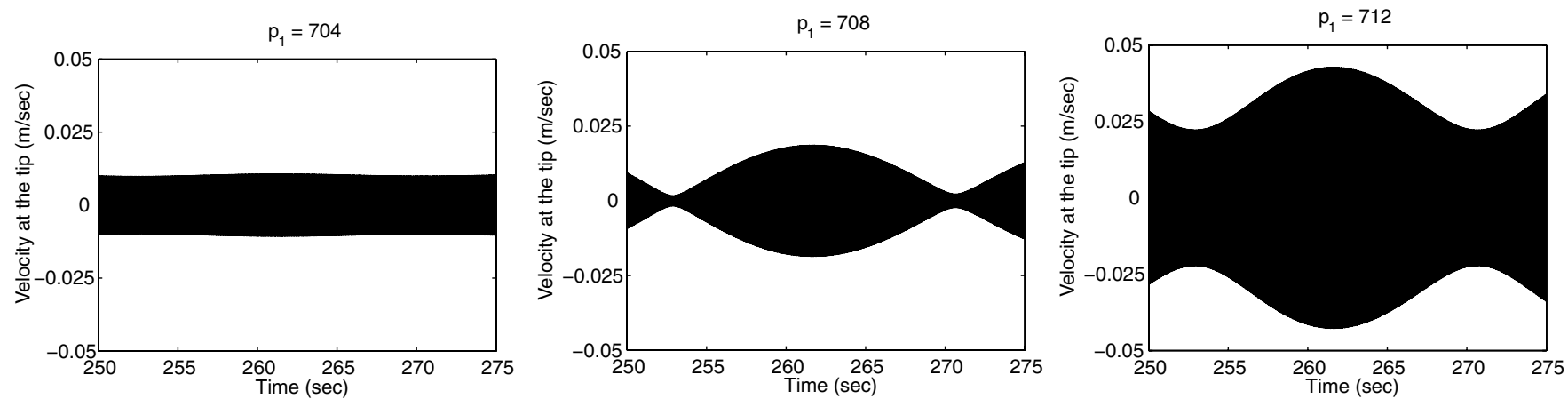

Fig. 4 Time response of velocity at the tip of the sensing beam without added mass for different values of $p_{1}$ (computational results).

$$
\begin{gathered}
E_{L}\left(p_{1}\right)= \begin{cases}A_{l} & \text { if } p_{1}<c_{5} \\
\left|A_{l}-A_{n}\right| & \text { if } p_{1} \geq c_{5}\end{cases} \\
= \begin{cases}\frac{1}{\sqrt{c_{1}+c_{2}\left(c_{3}-p_{1}\right)^{2}}} & \text { if } p_{1}<c_{5} \\
\left|\frac{1}{\sqrt{c_{1}+c_{2}\left(c_{3}-p_{1}\right)^{2}}}-c_{4} \sqrt{p_{1}-c_{5}}\right| & \text { if } p_{1} \geq c_{5}\end{cases} \\
E_{U}\left(p_{1}\right)= \begin{cases}A_{l} & \text { if } p_{1}<c_{5} \\
A_{l}+A_{n} & \text { if } p_{1} \geq c_{5}\end{cases} \\
= \begin{cases}\frac{1}{\sqrt{c_{1}+c_{2}\left(c_{3}-p_{1}\right)^{2}}} & \text { if } p_{1}<c_{5} \\
\frac{1}{\sqrt{c_{1}+c_{2}\left(c_{3}-p_{1}\right)^{2}}}+c_{4} \sqrt{p_{1}-c_{5}} & \text { if } p_{1} \geq c_{5}\end{cases}
\end{gathered}
$$

Figure 4 shows the time response of velocity at the tip of the beam without added mass for different values of $p_{1}$ (i.e., 704, 708, and 712). The three plots in Fig. 4 represent the dynamics for the bifurcation point, the beating response, and the postbeating response. Herein, the value of the controlled parameter $p_{1}$ at which Hopf bifurcation and the beating take place may be regarded as a quantitative indicator for detecting changes in system parameters such as mass. For example, in Fig. 3, the value of $p_{1}$ at which the bifurcation and beating occur varies from 703 to 713 and from 708 to 720 , due to different amounts of added mass.

Next, experimental results are discussed. Different amounts of mass (i.e., 10, 20, and $40 \mathrm{mg}$ ) are added at the tip of the sensing beam to create variations in the mass of the beam. Herein, the use of mass is due to the ease of removing it from and attaching it to the beam without changing the condition of the baseline case (i.e., no added mass). Then the controller circuits embedded in the real-time processor can be programmed such that the output analog signal (voltage) has exactly the same form as Eq. (9). Similar to numerical simulations, the parameter $p_{1}$ is varied and the other parameters are fixed. Figure 5 shows bifurcation diagrams resulting from experiments for the cases in which different amounts of mass (no mass, 20, and $40 \mathrm{mg}$ ) are added at the tip of the beam, and the parameters $p_{2}, p_{3}$, and $\Omega$ are specified as 4000,1 , and 118.12 $(18.8 \mathrm{~Hz})$.
One may note that the morphing of the bifurcation diagram in Fig. $\underline{5}$ due to added mass is qualitatively similar to the numerical results. For example, the value of the parameter $p_{1}$ corresponding to the dynamics with the minimum local amplitude shifts from left $\left(p_{1}=1.5\right)$ to right $\left(p_{1}=2.2\right)$. Also, for larger $p_{1}$ than each of these particular values, the width of the distribution of local amplitude maximum increases when the amount of mass increases. Furthermore, we applied changes to the value of the frequency $\Omega$ of the harmonic function in the controller to explore its influences on the bifurcation morphing. Figure 6 shows bifurcation diagrams obtained when the parameter $\Omega$ is specified as $118.75(18.9 \mathrm{~Hz})$. One may note that the bifurcation diagrams in Fig. 6 are more sensitive to variations in mass than those in Fig. 5. This is because the frequency used (of $18.9 \mathrm{~Hz}$ ) is closer to the fundamental frequency of the sensing beam. Note, however, that the fundamental frequency cannot be approached too closely to gain higher sensitivity because the output signal surpasses the maximum output voltage limit of the hardware. Finally, the values of the parameters leading to the dynamics with the minimum local amplitude attract our attention because they can be considered as the transition points from simple to complex limit cycles. Therefore, we investigated the movement of these transition points (caused by added mass at the tip) in the parameter space spanned by $\Omega$ and $p_{1}$.

Of course, the expressions for the amplitudes of the responses in Eqs. (10-12) are only qualitatively representative because, in general, superposition does not hold, and the components of the response do not simply add. Surprisingly, however, the simple qualitative estimation for the response is quite accurate, as shown in Figs. $3, \underline{5}$, and 6 . The envelopes $E_{L}$ and $E_{U}$ for the responses shown in the left plots of these figures are remarkably close to the accurate experimental and numerical results, which indicates that the beating phenomenon is the likely cause for the particular shape of the bifurcation diagrams and that the monitored value for $p_{1}$ corresponds to the situation in which $A_{l} \approx A_{n}$ and, simultaneously, $\Omega \approx \Omega_{n}$.

The envelope plotted in Fig. 3 was obtained using $c_{1}=8790$, $c_{2}=6.17, c_{3}=694, c_{4}=0.018$, and $c_{5}=707.5$. The values used in Fig. 5 were $c_{1}=45, c_{2}=0.29, c_{3}=6.54, c_{4}=0.34$, and $c_{5}=1.3$. Finally, the values used in Fig. $\underline{6}$ were $c_{1}=30, c_{2}=0.3$, $c_{3}=6.54, c_{4}=0.34$, and $c_{5}=1.4$.
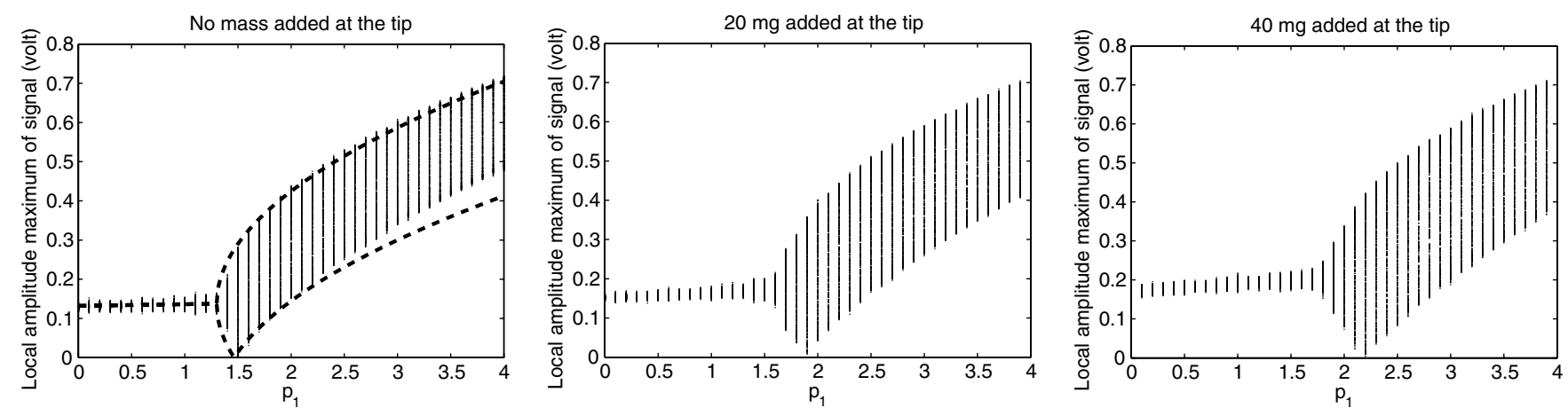

Fig. 5 Bifurcation diagrams for the cases in which different amounts of mass are added at the tip of the beam and the frequency of a harmonic function in the controller circuit is $18.8 \mathrm{~Hz}$ (experimental results). 

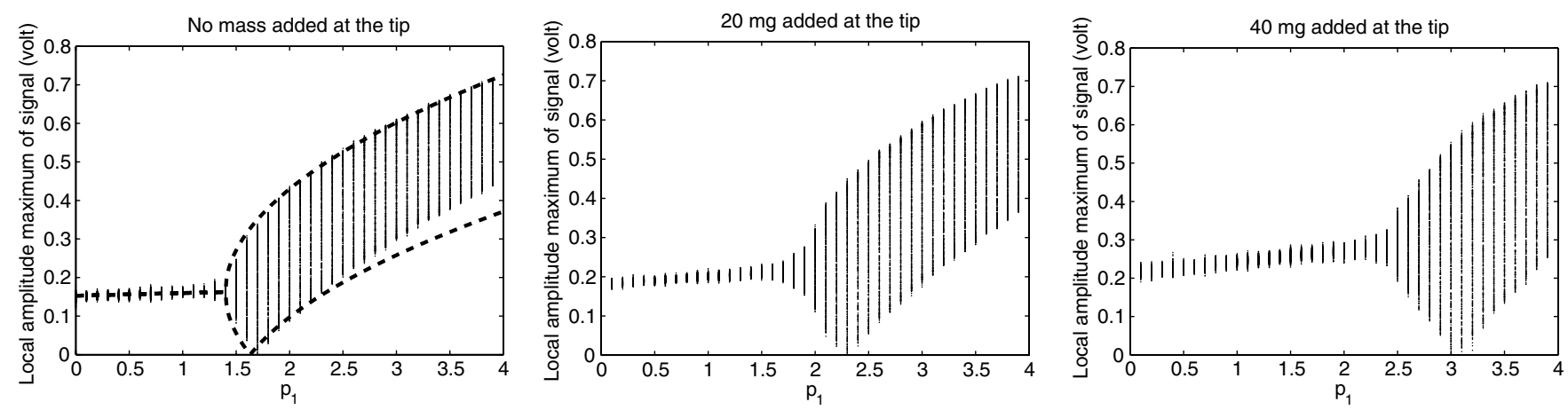

Fig. 6 Bifurcation diagrams for the cases in which different amounts of mass are added at the tip of the beam and the frequency of a harmonic function in the controller circuit is $18.9 \mathrm{~Hz}$ (experimental results).

Figures 7 and 8 show key results regarding the transition points in the parameter space for the cases in which different amounts of mass (i.e., no mass, 10, 20, and $40 \mathrm{mg}$ ) are added at the tip of the beam. The case in which no mass is added is considered as the baseline. The variations in the parameter $p_{1}$ with respect to the baseline due to different amounts of added mass for each specified frequency are shown in Fig. 8. From Fig. 8, one can notice that the variations in the parameter $p_{1}$ for the frequencies specified as 18.8 and $18.9 \mathrm{~Hz}$ are nearly proportional to the small amount of added mass (e.g., 10 and $20 \mathrm{mg}$ ). This proportionality provides an important basis for quantitatively predicting not only the presence of added mass, but also the amount of mass at the tip of the beam. Moreover, if the sensitivity for sensing the existence of added mass is more valued than the accuracy of predicting the amount of added mass, one may

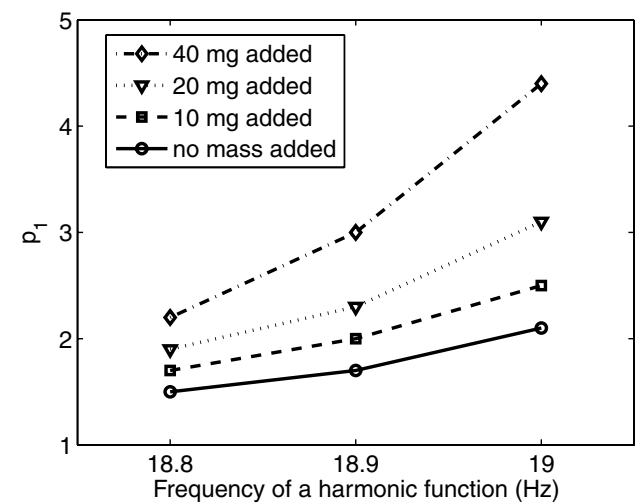

Fig. 7 Transition points in the parameter space for the cases in which different amounts of mass are added at the tip of the beam (experimental results).

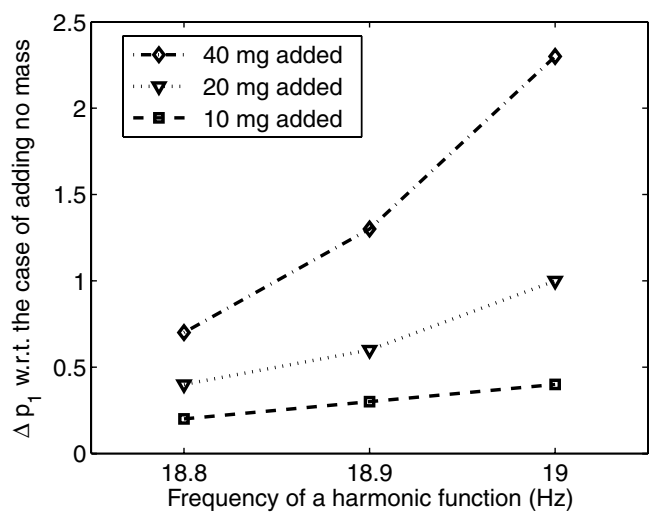

Fig. 8 Difference in the parameter $p_{1}$ with respect to the baseline (no added mass) due to different amounts of added mass for each specified frequency (experimental results). use a higher frequency (of $19 \mathrm{~Hz}$ ) to enhance sensitivity, as shown in Fig. 8 .

\section{Conclusions}

A novel method for identifying parameter variations based on active bifurcation morphing was demonstrated. To test this method experimentally, a cantilever (sensing) beam was built and excited by a nonlinear feedback excitation through two PZT patches bonded to the beam. The nonlinear feedback excitation was formed by a feedback loop, which consists of sending the analog signal of velocity measured from a laser vibrometer into a real-time processor, using this signal in a designed circuit in the processor, amplifying the output signal from the processor, and then applying the amplified voltage to the PZT patches.

One of the key challenges in this study was how to design the nonlinear controller (i.e., the nonlinear feedback excitation) such that bifurcation morphing would be very sensitive to small parameter variations. This problem was solved by employing a modified (driven) van der Pol oscillator and testing the form of the nonlinear feedback excitation by numerical simulations based on a finite element model of the sensing beam.

Both computational and experimental results showed that the sensitivity to small parameter variations (such as small changes in mass) can be significantly enhanced by the designed nonlinear feedback excitation. Also, the computational and experimental results showed that the bifurcation morphing modes can be used as features for damage detection and sensing.

The (functional form of the) nonlinear function used in the controller determines the type of bifurcation that is likely to be created by the nonlinear feedback excitation. Hence, this functional form does not directly depend on the system. However, the particular values of the coefficients of the linear and nonlinear terms used in the controller circuit depend on basic characteristics of the system, such as resonant frequencies. As a consequence, to implement the proposed approach, a mathematical model of the system is not needed per se, as long as a controller that creates bifurcations can be implemented. For example, in many applications, the controller could be designed based just on basic characteristics of the system, such as the fact that the system is linear with certain dominant natural frequencies (or known rough estimates for stiffness and mass, etc.) without a detailed model (e.g., an accurate/calibrated finite element method model). For example, to create the type of bifurcations employed in this paper, one could focus the excitation frequency near one of the resonant frequencies of the system and then (experimentally) tune the coefficients of the linear and cubic terms in a nonlinear feedback excitation to create beatinglike phenomena such as those observed in this work (e.g., Fig. 4). Next, parameter variations could be applied and the bifurcation boundary morphing could be tracked experimentally (as in a calibration procedure). Hence, the parameters that can be interrogated are not only mass, but could be others such as stiffness, and a detailed/accurate mathematical model of the system may not be needed. 
However, using the proposed approach to interrogate much more complex nonlinear systems than the beam used herein poses several challenges. These challenges are due to three main factors. First, like any other interrogation approach, one has to be able to inject enough energy in the dynamics to create significant motion, detectable accurately with the set of sensors employed. That poses nontrivial constraints on the type and location of the actuators and sensors that can be used. Second, one has to be able to apply a feedback excitation through a controller that is fast enough compared with the natural frequencies of the system. Large delays in the controller can lead to very complex dynamic behavior. Third, compared with the bifurcation induced in this work, creating other types of (desired) bifurcations to ensure high sensitivity for specific parameters of the system may not be trivial to achieve safely, especially in the case of more complex systems, with complex nonlinear behavior and mode coupling. Also, to our knowledge, which bifurcations are the best (i.e., the most sensitive) for monitoring specific parameter changes is unknown. Answering those issues is part of future work, beyond the scope of this paper.

\section{References}

[1] Doebling, S. W., Farrar, C. R., Prime, M. B., and Shevitz, D. W., "Damage Identification and Health Monitoring of Structural and Mechanical Systems from Changes in Their Vibration Characteristics: A Literature Review," Los Alamos National Labs., Rept. LA-13070MS, Los Alamos, NM, 1996.

[2] Farrar, C. R., Doebling, S. W., and Nix, D. A., "Vibration-Based Structural Damage Identification," Philosophical Transactions of the Royal Society of London, Series A: Mathematical and Physical Sciences, Vol. 359, No. 1778, 2001, pp. 131-149. doi:10.1098/rsta.2000.0717

[3] Abdalla, M. O., Grigoriadis, K. M., and Zimmerman, D. C., "Enhanced Structural Damage Detection Using Alternating Projection Methods," AIAA Journal, Vol. 36, No. 7, 1998, pp. 1305-1311.

[4] Pappa, R. P., James, G. H., and Zimmerman, D. C., "Autonomous Modal Identification of the Space Shuttle Tail Rudder," Journal of Spacecraft and Rockets, Vol. 35, No. 2, 1998, pp. 163-169.

[5] Zimmerman, D. C., "Model Validation and Verification of Large and Complex Space Structures," Inverse Problems in Engineering, Vol. 8, No. 2, 2000, pp. 93-118. doi: $10.1080 / 174159700088027722$

[6] D'Souza, K., and Epureanu, B. I., "Damage Detection in Nonlinear Systems Using System Augmentation and Generalized Minimum Rank Perturbation Theory," Smart Materials and Structures, Vol. 14, No. 5, 2005, pp. 989-1000. doi:10.1088/0964-1726/14/5/037

[7] Amizic, B., Amaravadi, V. K., Rao, V. S., and Derriso, M. M., "TwoDimensional Wavelet Mapping Techniques for Damage Detection in Structural Systems," Smart Structures and Materials 2002: Modeling, Signal Processing, and Control, edited by V. S. Rao, Proceedings of SPIE-The International Society for Optical Engineering, Vol. 4693, Society of Photo-Optical Instrumentation Engineers, Bellingham, WA, 2002, pp. 267-278.

[8] Amaravadi, V. K., Mitchell, K., Rao, V. S., and Derriso, M. M., "Structural Integrity Monitoring of Composite Patch Repairs Using Wavelet Analysis and Neural Networks," Smart Structures and Materials 2002: Smart Structures and Integrated Systems, edited by L. P. Davies, Proceedings of SPIE-The International Society for Optical Engineering, Vol. 4701, Society of Photo-Optical Instrumentation Engineers, Bellingham, WA, 2002, pp. 156-166.

[9] Cao, T. T., and Zimmerman, D. C., "Procedure to Extract Ritz Vectors from Dynamic Testing Data," Journal of Structural Engineering, Vol. 125, No. 12, 1999, pp. 1393-1400. doi:10.1061/(ASCE)0733-9445(1999)125:12(1393)

[10] Zimmerman, D. C., "Looking into the Crystal Ball: The Continued Need for Multiple Viewpoints in Damage Detection," Key Engineering Materials, Vols. 167-168, No. 1, 1999, pp. 76-90.

[11] Ray, L. R., and Tian, L., "Damage Detection in Smart Structures Through Sensitivity Enhancing Feedback Control," Journal of Sound and Vibration, Vol. 227, No. 5, 1999, pp. 987-1002. doi:10.1006/jsvi.1999.2392

[12] Ray, L. R., and Koh, B. H., "Enhancing Uniqueness Properties in Damage Identification Using Sensitivity Enhancing Control," Materials Evaluation, Vol. 61, No. 10, 2003, pp. 1134-1142.

[13] Koh, B. H., and Ray, L. R., "Localisation Of Damage in Smart
Structures Through Sensitivity Enhancing Feedback Control," Mechanical Systems and Signal Processing, Vol. 17, No. 4, 2003, pp. 837-855.

doi:10.1006/mssp.2003.1566

[14] Kim, J. S., Wang, K. W., and Smith, E. C., "High-Authority Piezoelectric Actuation System Synthesis Through Mechanical Resonance and Electrical Tailoring," Journal of Intelligent Material Systems and Structures, Vol. 16, No. 1, 2005, pp. 21-31. doi:10.1177/1045389X05046686

[15] Tang, J., and Wang, K. W., "Vibration Confinement via Optimal Eigenvector Assignment and Piezoelectric Networks," Journal of Vibration and Acoustics, Vol. 126, No. 1, 2004, pp. 27-36. doi: $10.1115 / 1.1597213$

[16] Tang, J., and Wang, K. W., "Vibration Delocalization of Nearly Periodic Structures Using Coupled Piezoelectric Networks," Journal of Vibration and Acoustics, Vol. 125, No. 1, 2003, pp. 95-108. doi:10.1115/1.1521951

[17] Nichols, J. M., Trickey, S. T., Todd, M. D., and Virgin, L. N., "Structural Health Monitoring Through Chaotic Interrogation," Meccanica, Vol. 38, No. 2, 2003, pp. 239-250. doi:10.1023/A:1022898403359

[18] Nichols, J. M., Todd, M. D., and White, J. R., "Using State Space Predictive Modeling with Chaotic Interrogation in Detecting Joint Preload Loss in a Frame Structure Experiment," Smart Materials and Structures, Vol. 12, No. 2, 2003, pp. 580-601.

[19] Nichols, J. M., Todd, M. D., Seaver, M., and Virgin, L. N., "Use of Chaotic Excitation and Attractor Property Analysis in Structural Health Monitoring," Physical Review E (Statistical Physics, Plasmas, Fluids, and Related Interdisciplinary Topics), Vol. 67, No. 016209, 2003, pp. 1-8.

[20] Nichols, J. M., Virgin, L. N., Todd, M. D., and Nichols, J. D., "On the Use of Attractor Dimension as a Feature in Structural Health Monitoring," Mechanical Systems and Signal Processing, Vol. 17, No. 6, 2003, pp. 1305-1320. doi:10.1006/mssp.2002.1521

[21] Trickey, S. T., Todd, M., Seaver, M., and Nichols, J., "Geometric TimeDomain Methods of Vibration-Based Damage Detection," Smart Nondestructive Evaluation for Health Monitoring of Structural and Biological Systems, Proceedings of SPIE-The International Society for Optical Engineering, Vol. 4702, Society of Photo-Optical Instrumentation Engineers, Bellingham, WA, 2002, pp. 113-121.

[22] Epureanu, B. I., and Yin, S. H., "Identification of Damage in an Aeroelastic System Based on Attractor Deformations," Computers and Structures, Vol. 82, No. 31-32, 2004, pp. 2743-2751. doi:10.1016/j.compstruc.2004.03.079

[23] Epureanu, B. I., Tang, L. S., and Paidoussis, M. P., "Exploiting Chaotic Dynamics for Detecting Parametric Variations in Aeroelastic Systems," AIAA Journal, Vol. 42, No. 4, 2004, pp. 728-735. doi:10.2514/1.9556

[24] Epureanu, B. I., Yin, S. H., and Derriso, M. M., "Attractor-Based Damage Detection in a Plate Subjected to Supersonic Flows," Health Monitoring and Smart Nondestructive Evaluation of Structural and Biological Systems 3, Proceedings of SPIE-The International Society for Optical Engineering, Vol. 5394, Society of Photo-Optical Instrumentation Engineers, Bellingham, WA, 2004, pp. 340-350.

[25] Epureanu, B. I., Yin, S. H., and Derriso, M. M., "High-Sensitivity Damage Detection Based on Enhanced Nonlinear Dynamics," Smart Materials and Structures, Vol. 14, No. 2, 2005, pp. 321-327. doi:10.1088/0964-1726/14/2/004

[26] Epureanu, B. I., Yin, S. H., and Dowell, E. H., "Enhanced Nonlinear Dynamics for Accurate Identification of Stiffness Loss in a ThermoShielding Panel," Nonlinear Dynamics, Vol. 39, No. 1-2, 2005 , pp. 197-211. doi:10.1007/s11071-005-1926-9

[27] Yin, S. H., and Epureanu, B. I., "Enhanced Nonlinear Dynamics and Monitoring Bifurcation Morphing for Structural Health Monitoring," 5th International Workshop on Structural Health Monitoring, Vol. 1, DEStech Publications, Lancaster, PA, 2005, pp. 1769-1776.

[28] Yin, S. H., and Epureanu, B. I., "Advanced System Interrogation Based on Active Bifurcation Morphing," 47th AIAA/ASME/ASCE/AHS/ ASC Structures, Structural Dynamics and Materials Conference, Newport, RI, AIAA Paper 2006-1884, 2006.

[29] Crawley, E. F., and Deluis, J., "Use of Piezoelectric Actuators as Elements of Intelligent Structures," AIAA Journal, Vol. 25, No. 10, 1987, pp. $1373-1385$.

[30] Hanagud, S., Obal, M. W., and Calise, A. J., "Optimal Vibration Control by the use of Piezoceramic Sensors and Actuators," Journal of Guidance, Control, and Dynamics, Vol. 15, No. 5, 1992, pp. 11991206. 
[31] Dosch, J., Leo, D., and Inman, D., "Modeling and Control for Vibration Suppression of a Flexible Active Structure," Journal of Guidance, Control, and Dynamics, Vol. 18, No. 2, 1995, pp. 340-346.

[32] Pai, P. F., Wen, B., Naser, A. S., and Schulz, M. J., "Structural Vibration Control Using PZT Patches and Non-Linear Phenomena," Journal of Sound and Vibration, Vol. 215, No. 2, 1998, pp. 273-296. doi:10.1006/jsvi.1998.1612

[33] Schulz, M. J., Pai, P. F., and Inman, D. J., "Health Monitoring and Active Control of Composite Structures Using Piezoceramic Patches," Composites, Part B, Vol. 30, No. 7, 1999, pp. 713-725. doi:10.1016/S1359-8368(99)00034-7
[34] Waanders, J. W., Piezoelectric Ceramics Properties and Applications, Philips Components, Eidenhoven, The Netherlands, 1991.

[35] Hwang, W. S., and Park, H. C., "Finite-Element Modeling of Piezoelectric Sensors and Actuators," AIAA Journal, Vol. 31, No. 5, 1993, pp. 930-937.

[36] Strogatz, S. H., Nonlinear Dynamics and Chaos, Addison-Wesley, Reading, MA, 1994.
M. Ahmadian Associate Editor 